\title{
The Modeling and Implementation of the Asset Management System of the Automobile Maintenance Shop Based on RFID
}

\author{
Yan Qi \\ College of Information Science and Technology, Bohai University, \\ Jinzhou, Liaoning, China \\ 32392392@qq.com
}

\begin{abstract}
The system can realize the information management, and help enterprises statistically analyze the information through the modern information technology, to guide the work of enterprises. The use of UML can do objectoriented analysis and modeling, creating a static mode for the systeml. RFID technology will be applied to the asset management system for auto repair shop, to achieve the positioning and management of assets, and to improve the efficiency of the car repair shop.
\end{abstract} C\#

Keywords-UML modeling; Maintenance management; RFID;

\section{INTRODUCTION}

Most of the car repair shop in China started late in the information construction. Even though there were computers, servers, network and other modern equipment, a lot of businesses were still done through manual operation, manual management, and paperless and modern office can not be realized in this case. The enterprise's work efficiency is low, and business process is complex. What's more, human and material resources waste is serious.

\section{UML MODELING}

System modeling is the core part of software development process. The purpose of modeling is to integrate the structure and behavior of the system, to better meet the needs of users.

The process of UML modeling is divided into three stages: requirement modeling, domain modeling and design implementation. The static modeling and dynamic modeling are two kinds of UML modeling mechanisms. Case diagram, class diagram and object diagram are used to define the relationship between the object and the static system in the system object model; The component diagram and deployment diagram reflect the software architecture[1], hardware architecture and communication mechanism; The state and interaction relationship between objects are described through sequence diagram, collaboration diagram, state diagram and activity diagram.

\section{A. Static modeling mechanism}

According to the functional requirements of the system in the process of analyzing and designing, we should firstly make the static modeling by UML, which is the foundation of the software model.The static modeling uses case diagram, class diagram, object diagram, package diagram, component diagram and configuration diagram.

\section{B. Dynamic modeling mechanism}

Dynamic modeling can reflect dynamic modeling mechanism through sequence diagram, collaboration diagram, state diagram and activity diagram. The interaction between objects is accomplished through the transmission of messages between objects, and the state of the object will change with the transmission results.

\section{NEEDS ANALYSIS}

Requirement analysis is the most basic and important part of software development. It is the premise of all the development and design. If you do not understand the needs of users and start design programming, no matter how the program is done, the consequences will be in vain. What is the basic task of requirements analysis is to tell us "what system must do?". Although we have a rough understanding of user's needs in the stage of feasibility analysis, feasibility study is done in a short period of time and at a lower cost to determine what work must be completed, and to show requirements in a complete, accurate, clear and specific way. The result of requirement analysis is the foundation of system development, which is related to the success or failure of the project and the quality of the software product.

\section{SYSTEM MODELING}

Case diagram is used to describe the operation of users or participants in the system, which plays an important role in the demand analysis stage. The whole development process of the system revolves around the problem and the model of the requirement.

\section{A. Overall business modeling}

The first step in creating a system case is to determine the system's participants, including system administrator, receptionist, purchaser, supplier, warehouse manager, maintenance staff, accounting[2], personnel manager and salesman after a comprehensive analysis of the system. Case 
package diagram of the system is shown in figure 1 through the above analysis .

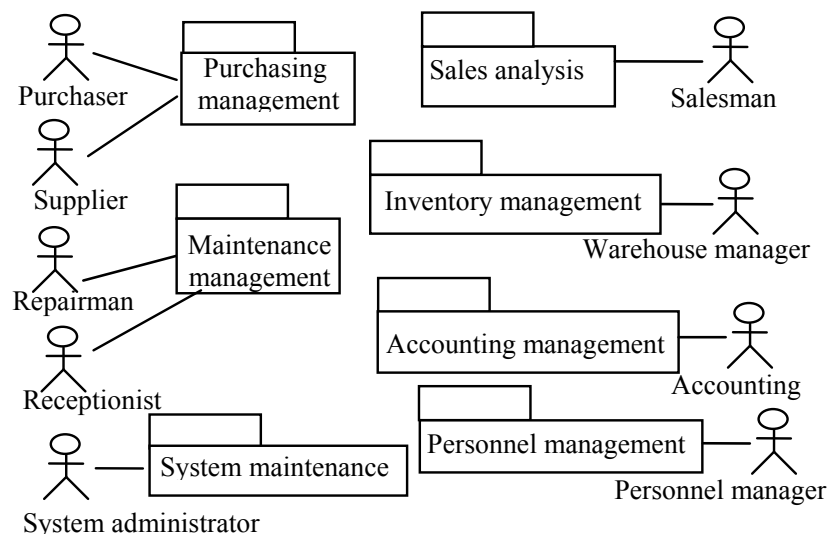

Fig. 1. Overall use case package

\section{B. Static modeling of inventory management module}

Inventory management is the core of the company's logistics management, whose main function is to be responsible for all products receiving, using, returning, and storing. Case diagram of inventory management is shown in Figure 2. Main business of inventory management is the product storage, inventory, inventory information query, loss and transfer operation[3].

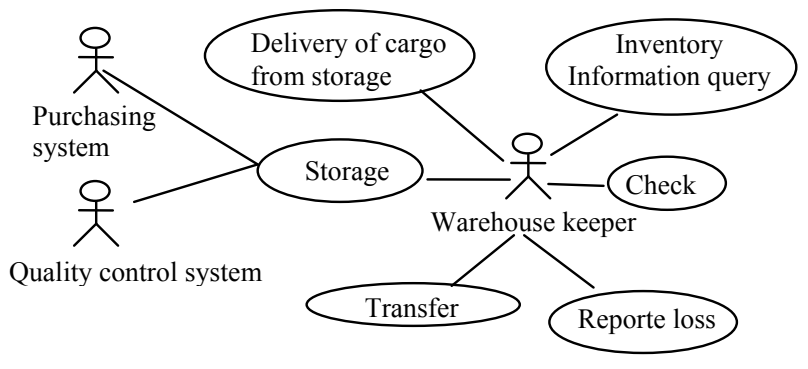

Fig. 2. Inventory management module use case diagram

\section{Maintenance management module static modeling}

The main function of maintenance management includes reception, dispatching, requisition, completion, settlement, cash and other operations. The case diagram of maintenance management is shown in figure 3. Front receptionist inputs customer information, equipment information and fault condition according to customer description, generates a maintenance order, and prints the maintenance order and confirms customer's signature. Detailed information of maintenance orders can be obtained through inputting query order number. After receiving the notice, maintenance staff begins to check the actual equipment failure, and calculate the need of accessories. If new problems in the process of maintenance is found, there may be possibilities of increasing accessories category and quantity, and new picking list will be submitted to the warehouse for the new parts. Maintenance staff can get requisition information through inputting the requisition number.

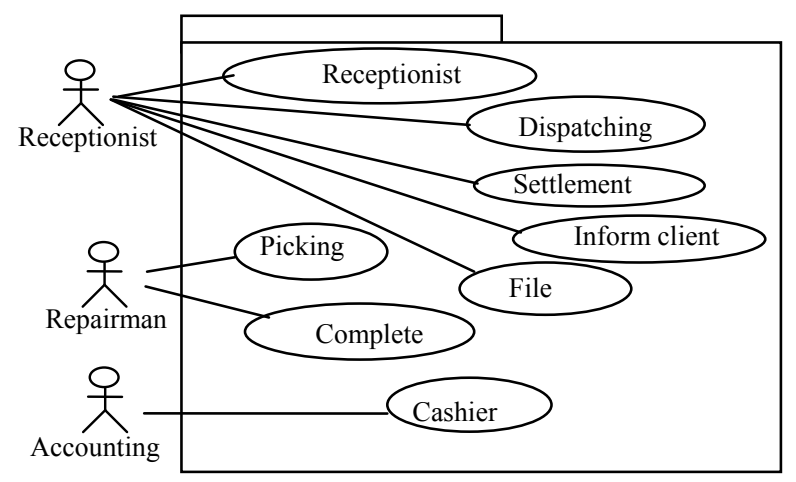

Fig. 3. Maintenance management use case diagram

\section{SYSTEM IMPLEMENTATION}

\section{A. Overall design}

Hardware use CS101 handheld RFID reader and the AD431 tag, the reading distance is 7 meters in the outdoor space, is 7-11 meters indoor space, the CS101 can read generally 150 tags per second. Software interface use C\# development, the background database is MySQL[4].

To set up Q Algorithm of the CS101 Reader, press "Setup" button in the Main Menu. Click on the tab "Inventory Config". In the field "Algorithm", choose "FIXEDQ" for Fixed Q Algorithm, "DYNAMICQ" for Dynamic Q Algorithm. System uses "DYNAMICQ".Press "Apply" button at the top right corner to confirm. The setting method is shown in figure 4.

\begin{tabular}{|c|c|c|c|c|}
\hline \multicolumn{5}{|c|}{ Apply } \\
\hline Gerenal Options & is Inventory & Config & Carrier Wa & 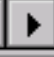 \\
\hline Operation & \multicolumn{2}{|c|}{$\checkmark$ Continuous } & \multicolumn{2}{|l|}{ Blocking mode } \\
\hline Selected & \multicolumn{2}{|l|}{$A L L$} & $\nabla$ & \\
\hline Session & \multicolumn{2}{|l|}{\begin{tabular}{|l} 
SO \\
FIXEDQ
\end{tabular}} & -2 & \\
\hline \multirow{2}{*}{ Target } & \multicolumn{2}{|l|}{ DYNAMICQ } & & \\
\hline & \multicolumn{2}{|l|}{ UNKNOWN } & & \\
\hline Algorithm & DYNAMICQ & & $\nabla$ & \\
\hline RSSI Filter & $\checkmark$ Enable & 75 & 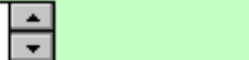 & \\
\hline
\end{tabular}

Fig. 4. DYNAMICQ settings

\section{B. System implementaion}

System implementation includes system interface design and processing, data storage, RFID tag reading and other operations. The following part focuses on the reading operation of RFID tag. Reading RFID tag information is a key 
part of system implementation. The indexes used are shown in Table 1.

TABLE I. CS101 API FUNCTION

\begin{tabular}{|l|l|l|}
\hline Function name & \multicolumn{1}{|c|}{$\begin{array}{c}\text { Return value } \\
\text { type }\end{array}$} & \multicolumn{1}{c|}{ Effect } \\
\hline StartupReader & Result & Start card reader \\
\hline ShutdownReader & Result & Close card reader \\
\hline Start & Result & Start operation \\
\hline Stop & Void & Close operation \\
\hline SetDynamicQParms & Result & Set Dynamic Q parameters \\
\hline RadioPowerOn & Bool & $\begin{array}{l}\text { Add power to the RFID } \\
\text { device }\end{array}$ \\
\hline RadioPowerOn & Bool & Close RFID device \\
\hline
\end{tabular}

When the system is running, the first step is to start RFID reader, turn on RadioPowerOn equipment, and then switch on StartupReader. There is abnormal situation if the return value is not rfid.Constants.Result.OK, which will lead to failure in reading the label operation[5].

Program code fragments are as follows:

RfidDe.RadioRowerOn();

If(Readde.StartupReader()!=rfid.Constants.Result.OK) \{

MessageBox.show(“ RFID device is fail!”);

Return;

\}

Using $($ Key=new KeyboardHook( $))$

\{

Application.Run(dengluform);

\}

Readde.ShutdownReader();

Device.RadioPowerOff();

We can decide to read labels or stop the read operation when the RFID reader is idle, and when it starts scanning. The first step is to set the DynamicQ parameters; The second step is to set the scan algorithm DYNAMICQ. The RunOnce attribute value is set to false.In the end, we can call the Start function to start the scanning operation, and call the Stop function to stop the scanning operation.

Program code fragments are as follows:

If(cx.Readerde.State $==$ ReaderOperationMode.Idle \&\&(B uttonState)bn_check.Tag==ButtonState.Start)

\{

bn_check.Tag=ButtonState.Stop;

bn_check.Text="stop";

cx.Readerde.SetDynamicQParms $(5,0,15,0,10,1)$;

cx.Readerde.RdrOpParms.RunSerachAnyparms.Method=Sing ulationAlgorithm.DYNAMICQ;

cx.Readerde.RdrOpParms.RunSerachAnyparms.RunOnc $\mathrm{e}=$ false;

cx.Readerde.RdrOpParms.Operation=Reader.Constants.

Operation.SearchAnyTags;

cx.Readerde.Start();

\}

Else

\{

bn_check.Tag=ButtonState.Start;
bn_check.Text="verify" ;

cx.Readerde.stop;

\}

CallBack function codes are as follows when CS101 finds a new Tag.

updateInvUI(e.InventoryInformation);

Private delegate void UpdateInvUIDeleg(InvertoryDataStruct InventryInformation);

Private void UpdateInvUI(InvertoryDataStruct

InventryInformation)

\{

If(this.InvokeRequired)

this.BeginInvoe(new

updateInvUIDeleg(UpdateInvUI)),new

object[]\{InventoryInformation $\})$;

for(int $\mathrm{i}=0 ; \mathrm{i}<$ assetlistView.Items.Count $; \mathrm{i}++$ )

\{

ListViewItem item=assetlistView.Items[i];

If(item.subItems[2].Text.Equals(InventryInformation.EPC

.ToString()))

\{

Item.SubItems[10].Text=“ok";

Item.BackColor=Color.Blue;

Return;

\})

assetlistView.Update();

\}

\section{CONCLUSION}

System modeling is the core of software development process. The purpose of modeling is to combine designed structure with system behavior to better meet the needs of users. This paper firstly analyzes the requirements of system function. Taking MySql as the background database, the author realizes automatic management of automobile parts, and improves the working efficiency of auto maintenance shop by using the UML static model, C\# programming, and RFID technology.

\section{REFERENCES}

[1] Mohammad ALSHAYEB; Nasser KHASHAN; Sajjad MAHMOOD, “A framework for an integrated unified modeling language, " Frontiers of Information Technology \& Electronic Engineering[j], vol. 38,pp.143159. 2016.

[2] Boulil, K., Bimonte, S., Pinet, F, "Conceptual model forspatial data cubes: a UML profile and its automaticimplementation," Comput. Stand. Interf, vol. 38,pp.113-132. 2015.

[3] Ye F,hu X D,ang Y G, "New Software MaintainabilityEvaluation Model Based on Multiple Classifiers Combination[C]. "International Conference on Qaulity,Reliability, $\mathrm{R}$ isk,Maintenance, and Safety Engineering,Chengdu,China,pp.321-325.2013.

[4] DING Yi, HU Jianguo, DUAN Zhikui, WANG Deming, DING Yanyu and TAN Hongzhou , " Built-in ESD Protection for RFID Tag ICs", Chinese Journal of Electronics.China,Vol.25, No.6, pp.10541062.vember 2016.

[5] Bei Yi Jun, The application of RFID technology in the Internet of things, the posts and Telecommunications Press.China,pp. 177-188. August 2014. 